\title{
Analysis of mechanisms with flexible beam-like links, rotary joints and assembly errors
}

Received: 16 September 2010 / Accepted: 1 June 2011 / Published online: 25 June 2011

(C) The Author(s) 2011. This article is published with open access at Springerlink.com

\begin{abstract}
The paper presents a method of modelling dynamics of mechanisms in which assembly errors can occur. One of the features of the method is that such errors can be included when the kinematics of the mechanism is modelled. A closed kinematic chain consisting of flexible and rigid links with one joint displaced and turned is discussed. Use of joint coordinates together with the rigid finite element method for discretisation of flexible links has allowed us to considerably decrease the size of the problem. Numerical simulations are carried out in order to analyse the influence of inaccurate assembly on the load on joints of a four-bar linkage.
\end{abstract}

Keywords Dynamic analysis $\cdot$ Mechanisms $\cdot$ Joint coordinates $\cdot$ Flexible links $\cdot$ Rigid finite element method

\section{Introduction}

For most research dealing with modelling dynamics of mechanisms, it is assumed that links and joints that connect the links are manufactured without deviations. In real systems, this assumption can be fulfilled only approximately. Inaccuracies of the technological process can play a decisive role. A mechanism whose links have been manufactured with errors requires additional forces during assembly process. This is a reason that some links may be deformed and additional loads can occur in joints. Reference [5] presents a method of kinetostatic analysis of overconstrained mechanisms with redundant joints. In this analysis, dimensional errors of the links are considered. The displacement matrix method has been used to evaluate internal loads of mechanisms. Pott and co-authors of Ref. [6] investigated the influence of dimensional errors of links on the position and orientation of the end-effector of a manipulator and Linapod parallel mechanism. Errors occurring during assembly may be another reason for inaccuracy in mechanisms and thus additional loads. In Ref. [7], assembly and manufacturing errors are treated as a kind of disturbance, which is then included in the mathematical model. Our paper presents a method of dynamic analysis of spatial mechanisms with flexible links connected by rotary joints, which takes into account inaccuracy of assembly. In the mathematical model presented, assembly errors are included in the description of the kinematics of the mechanism. Joint coordinates and homogenous transformations are used for deriving the model.

\section{Mathematical model of the mechanisms}

A closed kinematic chain consisting of $N$ rigid or flexible links as in Fig. 1 is considered. All links are connected by means of rotary joints. Ideally, namely when there are no assembly errors, axes of joints are parallel and the mechanism performs plane motion. As a result of inaccurate assembly, the joint connecting the last

K. Augustynek · I. Adamiec-Wójcik ( $\varangle)$

Department of Transport and Informatics, University of Bielsko-Biała, Bielsko-Biała, Poland

E-mail: i.adamiec@ath.bielsko.pl 


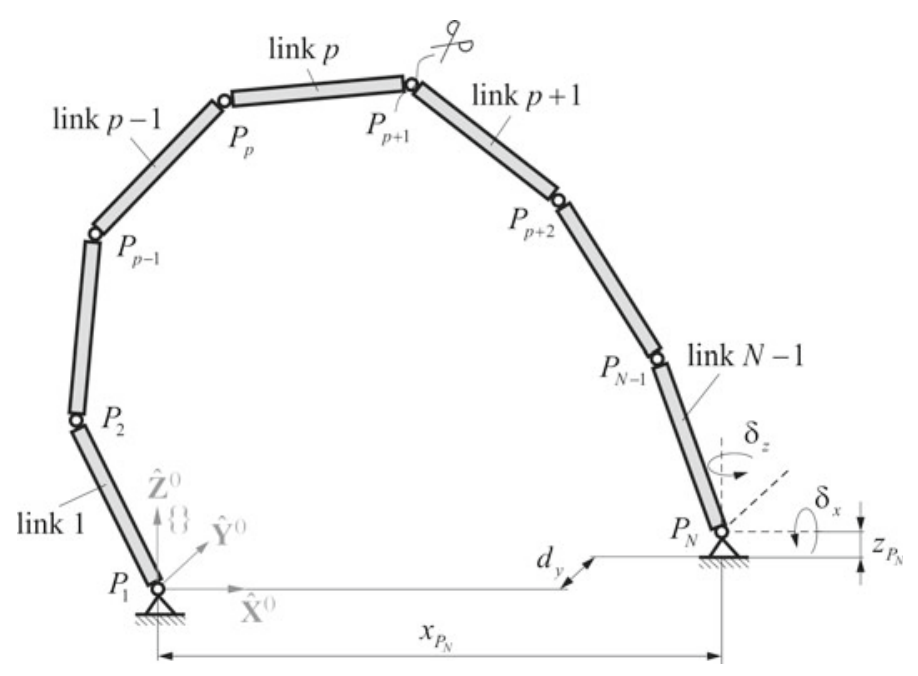

Fig. 1 Mechanism with assembly errors

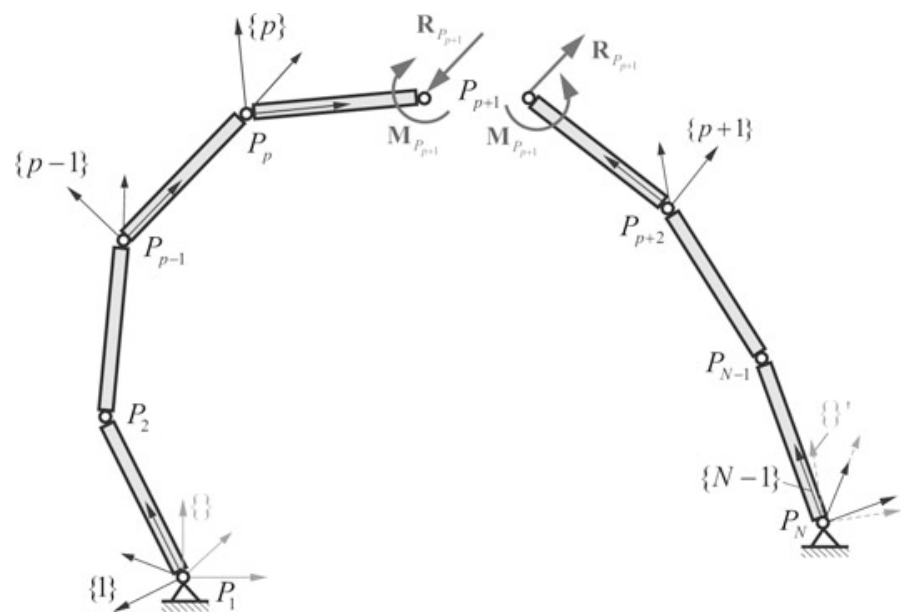

Fig. 2 An equivalent open kinematic chain

link with the base is shifted along axis $\hat{\mathbf{Y}}^{0}$ for distance $d_{y}$ and rotated about axes $\hat{\mathbf{Z}}^{0}$ and $\hat{\mathbf{X}}^{0}$ of the global system about angles $\delta_{z}$ and $\delta_{x}$, respectively. Thus, the mechanism becomes a spatial system, and additional forces and moments occur.

\subsection{Kinematics of the mechanism with assembly errors}

In order to describe the motion of the mechanism in joint coordinates, a virtual cut has to be made in the closed kinematic chain. The place at which it is done is arbitrary. It can be chosen so that the final kinematic chains are the shortest possible. Having cut the kinematic chain, a supplementary open system is obtained and additional reaction forces are introduced. Coordinate systems are assigned to each link in the way presented in Fig. 2.

Flexible links are discretised using the classical rigid finite element method described in detail in Ref. [8]. As a result, flexible link $p$ is replaced by a system of $m^{(p)}+1$ rigid finite elements (rfe) connected by means of $m^{(p)}$ massless and non-dimensional spring-damping elements (sde) (Fig. 3). Two coordinate systems are assigned to each rfe. System $\{p, i\}$ is placed at the centre of mass, and its axes coincide with central inertial axes. This system moves with the element, while system $\{p, i\}_{u}$, which has the same position as $\{p, i\}$ before deformation of the link, stays fixed. 


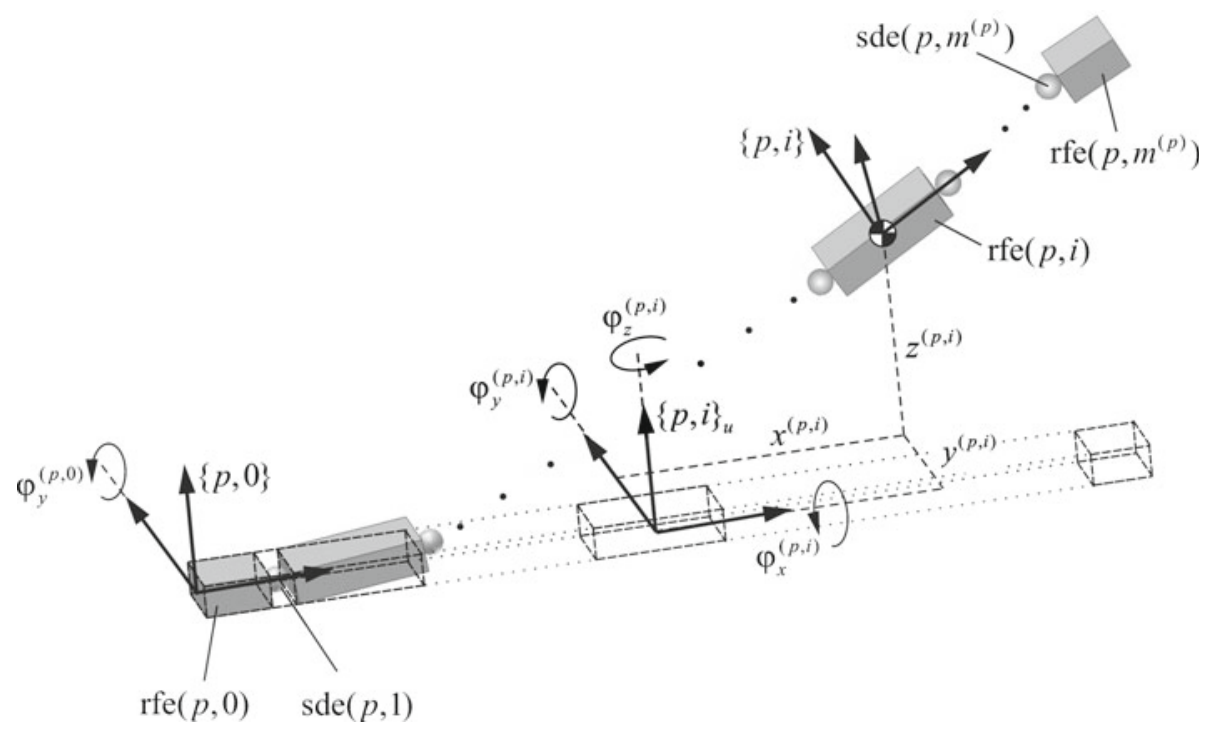

Fig. 3 Discretisation of flexible link $p$

Each element may have up to six degrees of freedom. The motion of rfe $(p, i)$ with respect to its position in the undeformed state is defined by the following vector of generalised coordinates:

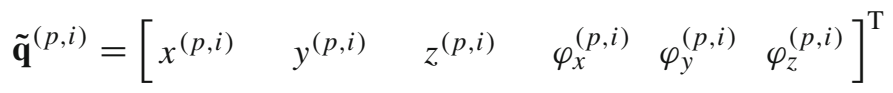

where $i=1, \ldots, m^{(p)}$.

Transformation of coordinates from system $\{p, i\}$ to system $\{p, 0\}$ can be written as:

$$
\tilde{\mathbf{B}}^{(p, i)}=\tilde{\mathbf{B}}_{u}^{(p, i)} \tilde{\mathbf{B}}_{d}^{(p, i)}
$$

where $\tilde{\mathbf{B}}_{u}^{(p, i)}$ is a constant transformation matrix from system $\{p, i\}_{u}$ assigned to $\operatorname{rfe}(p, i)$ in the undeformed state to system $\{p, 0\}$ and $\tilde{\mathbf{B}}^{(p, i)}$ defines position and orientation of $\operatorname{rfe}(p, i)$ after deformation with respect to coordinate system $\{p, i\}_{u}$.

In the case when link $p$ is not initially curved, the axes of $\{p, i\}_{u}$ are parallel to the axes of system $\{p, 0\}$, and the transformation matrix takes the form:

$$
\tilde{\mathbf{B}}_{u}^{(p, i)}=\left[\begin{array}{ll}
\mathbf{I} & \tilde{\mathbf{x}}_{0}^{(p, i)} \\
\mathbf{0} & 1
\end{array}\right]
$$

where $\mathbf{I}$ is a unity matrix and $\tilde{\mathbf{x}}_{0}^{(p, i)}$ describes the position of $\operatorname{rfe}(p, i)$ with respect to $\operatorname{rfe}(p, 0)$ before deformation.

Matrix $\tilde{\mathbf{B}}_{d}^{(p, i)}$ defines the coordinate transformation from system $\{p, i\}$ to $\{p, i\}_{u}$ as follows:

$$
\tilde{\mathbf{B}}_{d}^{(p, i)}=\left[\begin{array}{llll}
c_{z}^{(p, i)} c_{y}^{(p, i)} & c_{z}^{(p, i)} s_{y}^{(p, i)} s_{x}^{(p, i)}-s_{z}^{(p, i)} c_{x}^{(p, i)} & c_{z}^{(p, i)} s_{y}^{(p, i)} s_{x}^{(p, i)}+s_{z}^{(p, i)} s_{x}^{(p, i)} & x^{(p, i)} \\
s_{z}^{(p, i)} c_{y}^{(p, i)} & s_{z}^{(p, i)} s_{y}^{(p, i)} s_{x}^{(p, i)}+c_{z}^{(p, i)} c_{x}^{(p, i)} & s_{z}^{(p, i)} s_{y}^{(p, i)} c_{x}^{(p, i)}-c_{z}^{(p, i)} s_{x}^{(p, i)} & y^{(p, i)} \\
-s_{y}^{(p, i)} & c_{y}^{(p, i)} s_{x}^{(p, i)} & c_{y}^{(p, i)} c_{x}^{(p, i)} & z^{(p, i)} \\
0 & 0 & 0 & 1
\end{array}\right]
$$

where $c_{\xi}^{(p, i)}=\cos \varphi_{\xi}^{(p, i)}$ and $s_{\xi}^{(p, i)}=\sin \varphi_{\xi}^{(p, i)}, \xi \in\{x, y, z\}$.

It is assumed that link $p$ is connected with the end of preceding link $p-1$ by $\operatorname{rfe}(p, 0)$. In the case when link $p-1$ is flexible, $\operatorname{rfe}\left(p-1, m^{(p-1)}\right)$ is preceding link $p$. Degrees of freedom of the joint connecting $\operatorname{rfe}(p, 0)$ with the preceding link are its generalised coordinates. For the rotary joint, we can write:

$$
\tilde{\mathbf{q}}^{(p, 0)}=\left[\varphi_{y}^{(p, 0)}\right]
$$


Transformation of coordinates from $\{p, 0\}$ to $\{p-1\}$ assigned to the preceding link is defined by the matrix:

$$
\tilde{\mathbf{B}}^{(p, 0)}=\left[\begin{array}{ll}
\tilde{\mathbf{R}}^{(p, 0)} & \tilde{\mathbf{x}}^{(p, 0)} \\
\mathbf{0} & 1
\end{array}\right]
$$

where $\tilde{\mathbf{R}}^{(p, 0)}=\tilde{\mathbf{R}}^{(p, 0)}\left(\varphi_{y}^{(p, 0)}\right)$ is a rotation matrix,

$$
\tilde{\mathbf{R}}^{(p, 0)}=\left[\begin{array}{lll}
c_{y}^{(p, 0)} & 0 & s_{y}^{(p, 0)} \\
0 & 1 & 0 \\
-s_{y}^{(p, 0)} & 0 & c_{y}^{(p, 0)}
\end{array}\right], \quad c_{y}^{(p, 0)}=\cos \varphi_{y}^{(p, 0)}, \quad s_{y}^{(p, 0)}=\sin \varphi_{y}^{(p, 0)},
$$

$\tilde{\mathbf{x}}^{(p, 0)}$ defines the position of the origin of $\{p, 0\}$ with respect to $\{p-1\}$.

The rigid finite element method treats a rigid link as a special case of a flexible link, which consists of only $\operatorname{rfe}(p, 0)$. Thus, the following takes place:

$$
\begin{aligned}
\tilde{\mathbf{B}}^{(p)} & =\tilde{\mathbf{B}}^{(p, 0)} \\
\tilde{\mathbf{q}}^{(p)} & =\tilde{\mathbf{q}}^{(p, 0)}
\end{aligned}
$$

where $\tilde{\mathbf{B}}^{(p)}$ and $\tilde{\mathbf{q}}^{(p)}$ are the transformation matrix and the vector of generalised coordinates describing the relative motion of rigid link $p$ with respect to $p-1$. When assembly errors occur in the mechanism, an additional coordinate system \{\}$^{\prime}$ is defined (Fig. 2). Its origin is placed at point $P_{N}$ and turned about the inertial system according to the assembly errors. The respective transformation matrix to inertial system \{\} is defined as:

$$
\mathbf{A}^{(N-1)}=\left[\begin{array}{llll}
c_{\delta_{z}} & -s_{\delta_{z}} c_{\delta_{x}} & s_{\delta_{z}} s_{\delta_{x}} & x_{P_{N}} \\
s_{\delta_{z}} & c_{\delta_{z}} c_{\delta_{x}} & -c_{\delta_{z}} s_{\delta_{x}} & d_{y} \\
0 & s_{\delta_{x}} & c_{\delta_{x}} & z P_{N} \\
0 & 0 & 0 & 1
\end{array}\right]
$$

where $c_{\delta_{\xi}}=\cos \delta_{\xi}$ and $s_{\delta_{\xi}}=\sin \delta_{\xi}, \xi \in\{x, z\}$.

The motion of link $N-1$ is defined with respect to intermediate system \{\}$^{\prime}$. When the mechanism is ideal, this system is parallel to the inertial system. In the general case, the transformation matrix can be calculated using the following algorithm:

(1) assume $\mathbf{A}^{(1)}=\mathbf{I}$ and calculate $\mathbf{A}^{(N-1)}$ according to formula (8),

(2) for link $k=1,2, \ldots, p$

(2.1) evaluate local transformation matrix $\tilde{\mathbf{B}}^{(k, 0)}$ using formula (6)

(2.1) calculate global transformation matrix $\mathbf{B}^{(k, 0)}=\mathbf{A}^{(k)} \tilde{\mathbf{B}}^{(k, 0)}$

(2.2) for $\mathrm{rfe} i=1,2, \ldots, m^{(k)}$

(2.2.1) evaluate local transformation matrix $\tilde{\mathbf{B}}^{(k, i)}$ of the rfe $(k, i)$ using formula (2)

(2.2.2) calculate global transformation matrix from $\mathbf{B}^{(k, i)}=\mathbf{B}^{(k, 0)} \tilde{\mathbf{B}}^{(k, i)}$,

(2.4) assign $\mathbf{A}^{(k+1)}=\mathbf{B}^{\left(k, m^{(k)}\right)}$,

(3) for link $k=N-1, N-2, \ldots, p+1$

(3.1) evaluate local transformation matrix $\tilde{\mathbf{B}}^{(k, 0)}$ using formula (6)

(3.2) calculate global transformation matrix $\mathbf{B}^{(k, 0)}=\mathbf{A}^{(k)} \tilde{\mathbf{B}}^{(k, 0)}$

(3.3) for $\mathrm{rfe} i=1,2, \ldots, m^{(k)}$

(3.2.1) evaluate local transformation matrix $\tilde{\mathbf{B}}^{(k, i)}$ of the $\operatorname{rfe}(k, i)$ using formula (2)

(3.2.2) calculate global transformation matrix $\mathbf{B}^{(k, i)}=\mathbf{B}^{(k, 0)} \tilde{\mathbf{B}}^{(k, i)}$,

(3.4) $\quad \operatorname{assign} \mathbf{A}^{(k-1)}=\mathbf{B}^{\left(k, m^{(k)}\right)}$. 


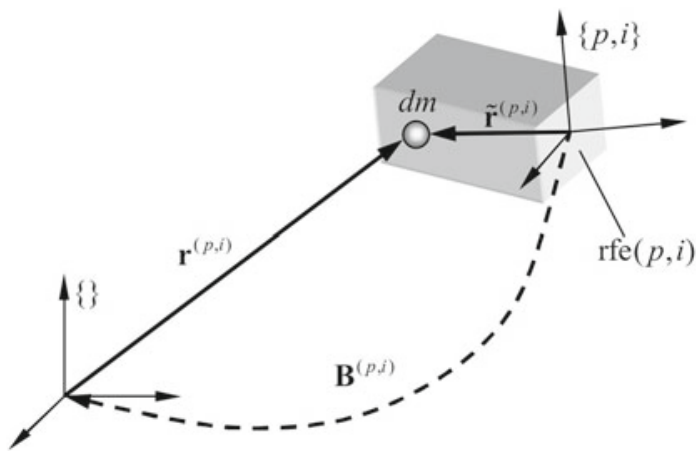

Fig. 4 Position of particle $\mathrm{d} m$ of the $\operatorname{re}(p, i)$

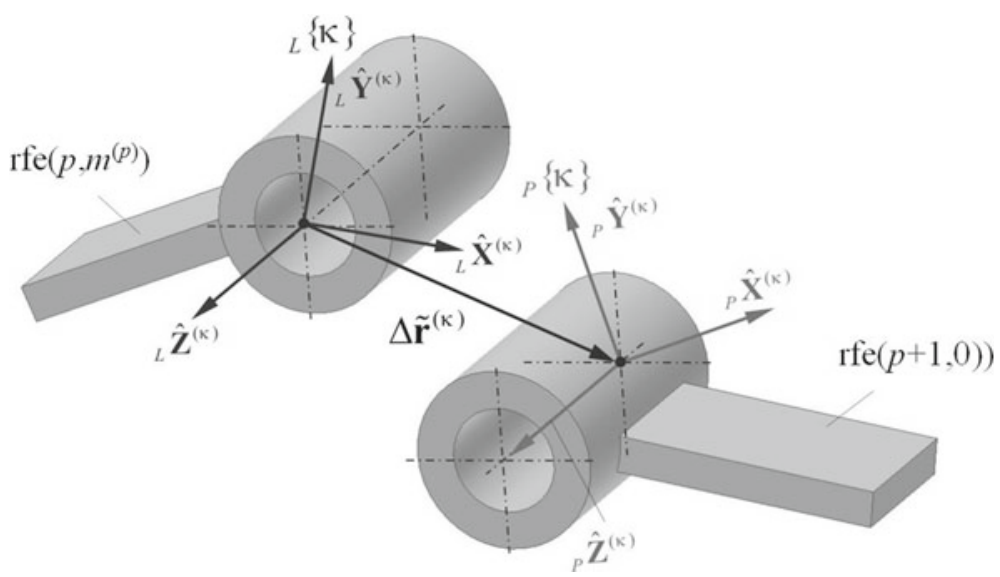

Fig. 5 Model of a rotary joint

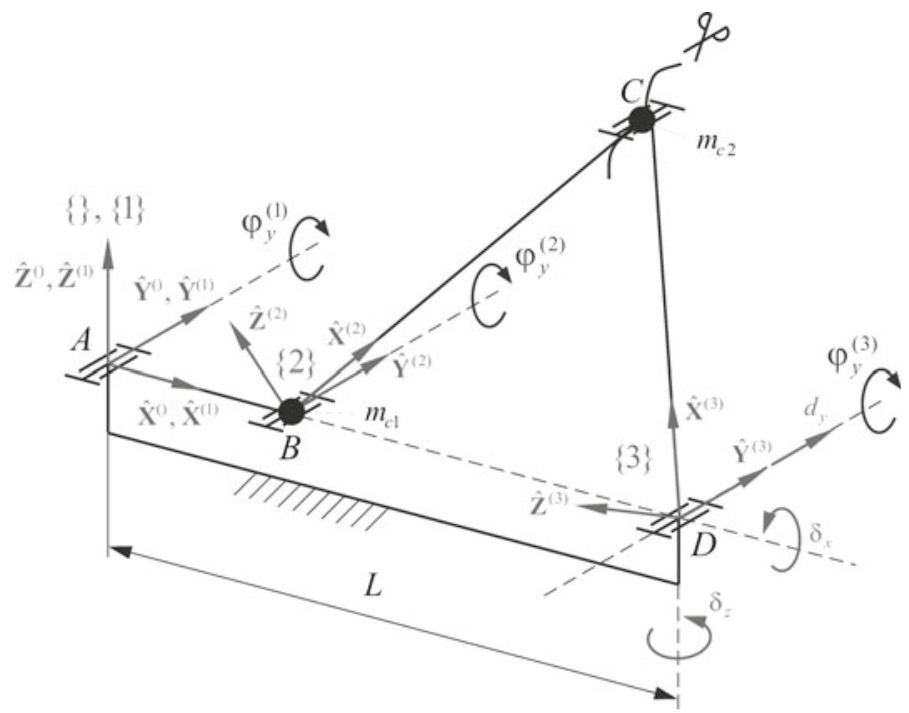

Fig. 6 Four-bar linkage mechanism 
2.2 Equations of motion of the mechanism

Equations of motion are derived from the Lagrange equations:

$$
\frac{\mathrm{d}}{\mathrm{d} t} \frac{\partial E}{\partial \dot{\mathbf{q}}}-\frac{\partial E}{\partial \mathbf{q}}+\frac{\partial V}{\partial \mathbf{q}}=\mathbf{Q}
$$

where $E, V$ are kinetic and potential energies of the mechanism and $\mathbf{Q}$ is the vector of generalised forces resulting from external loads. The vector of generalised coordinates $\mathbf{q}$ is defined as:

$$
\mathbf{q}=\left[\begin{array}{l}
\tilde{\mathbf{q}}^{(1)} \\
\vdots \\
\tilde{\mathbf{q}}^{(p)} \\
\vdots \\
\tilde{\mathbf{q}}^{(N)}
\end{array}\right]
$$

where $\tilde{\mathbf{q}}^{(p)}=\left[\begin{array}{lllll}\tilde{\mathbf{q}}(p, 0)^{T} & \ldots & \tilde{\mathbf{q}}(p, i)^{T} & \ldots & \tilde{\mathbf{q}}\left(p, m^{(p)}\right)^{T}\end{array}\right]^{T}$ is the vector of generalised coordinates of link $p$.

Kinetic energy $E$ is a sum of energies of all links of the mechanism:

$$
E=\sum_{p=1}^{N} \sum_{i=0}^{m^{(p)}} E^{(p, i)}
$$

where $E^{(p, i)}$ is the kinetic energy of $\operatorname{rfe}(p, i)$.

In order to derive the kinetic energy of $\operatorname{rfe}(p, i)$, let us consider particle $d m$ of $\operatorname{rfe}(p, i)$ (Fig. 4).

Coordinates of the particle in global coordinate system \{\} can be defined according to the formula:

$$
\mathbf{r}^{(p, i)}=\mathbf{B}^{(p, i)} \tilde{\mathbf{r}}^{(p, i)}
$$

Then, the kinetic energy of $\operatorname{rfe}(p, i)$ has a form:

$$
E^{(p, i)}=\int \frac{1}{2} \dot{\mathbf{r}}^{(p, i)^{T}} \dot{\mathbf{r}}^{(p, i)} \mathrm{d} m
$$

In order to derive the kinetic energy, the trace of a matrix is used [8]. Taking into account (12), the following can be written:

$$
\begin{aligned}
E^{(p, i)} & =\frac{1}{2} \int \operatorname{tr}\left\{\dot{\mathbf{r}}^{(p, i)} \dot{\mathbf{r}}^{(p, i)^{T}}\right\} \mathrm{d} m \\
& =\frac{1}{2} \int_{m^{(p, i)}} \operatorname{tr}\left\{\dot{\mathbf{B}}^{(p, i)} \tilde{\mathbf{r}}^{(p, i)} \tilde{\mathbf{r}}(p, i)^{T} \dot{\mathbf{B}}(p, i)^{T}\right\} \mathrm{d} m
\end{aligned}
$$

where $\operatorname{tr}\{\mathbf{A}\}=\sum_{i=1}^{m} a_{i i}, \mathbf{A}_{m \times m}=\left(a_{i, j}\right)_{i, j=1, \ldots, m}$-trace of a matrix, $\dot{\mathbf{B}}^{(p, i)}=\frac{\mathrm{d} \mathbf{B}^{(p, i)}}{\mathrm{d} t}$. 
Table 1 Cases of calculations for the assembly errors

\begin{tabular}{llll}
\hline Case & $\delta_{x}\left(^{\circ}\right)$ & $\delta_{z}\left({ }^{\circ}\right)$ & $d_{y}(\mathrm{~mm})$ \\
\hline P1 & 0 & 0 & 0 \\
P2 & 1 & 0 & 0 \\
P3 & 0 & 1 & 0 \\
P4 & 0 & 0 & 1 \\
P5 & 1 & 1 & 1 \\
\hline
\end{tabular}
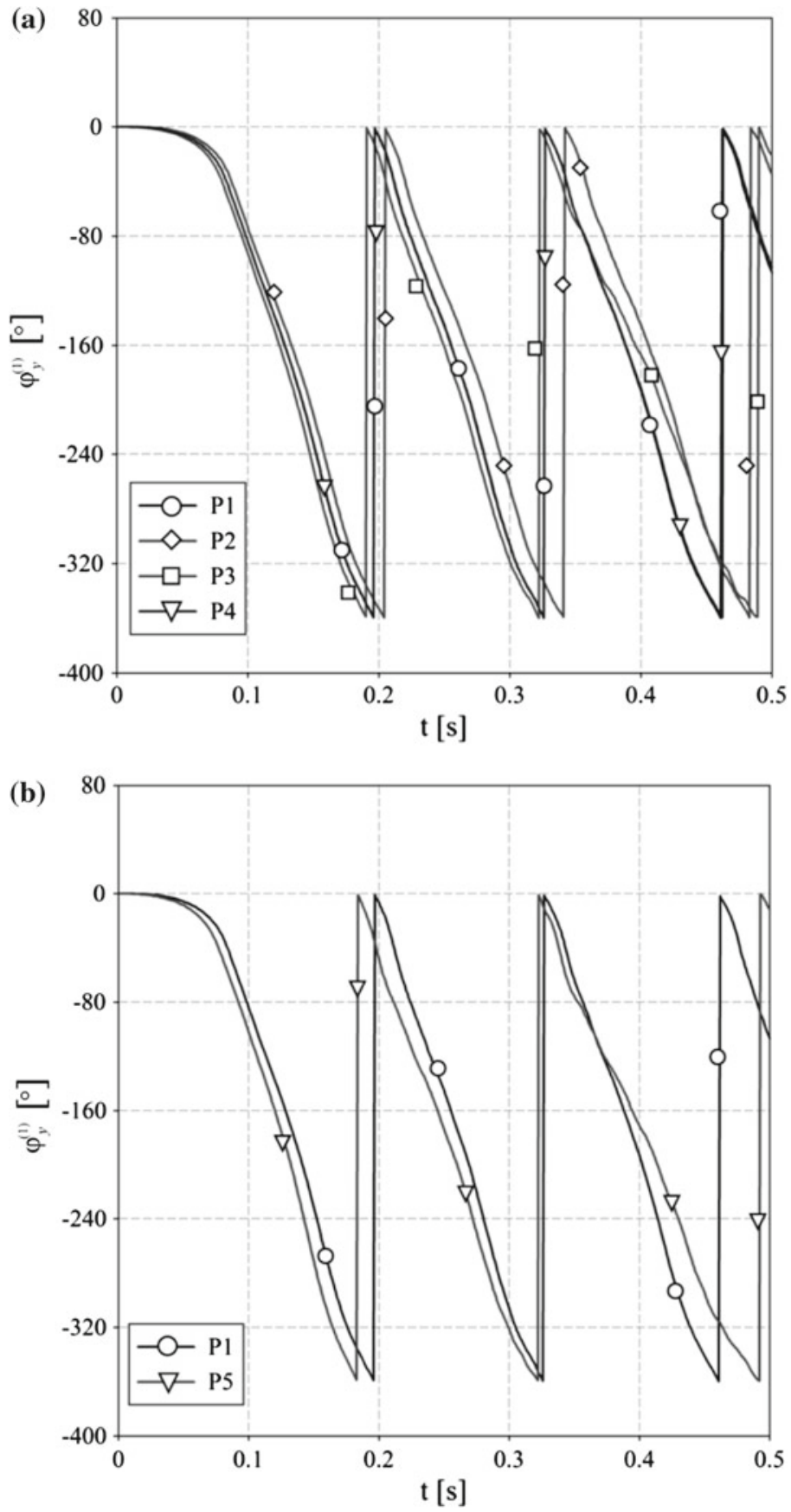

Fig. 7 Courses of rotation angle of the crank 

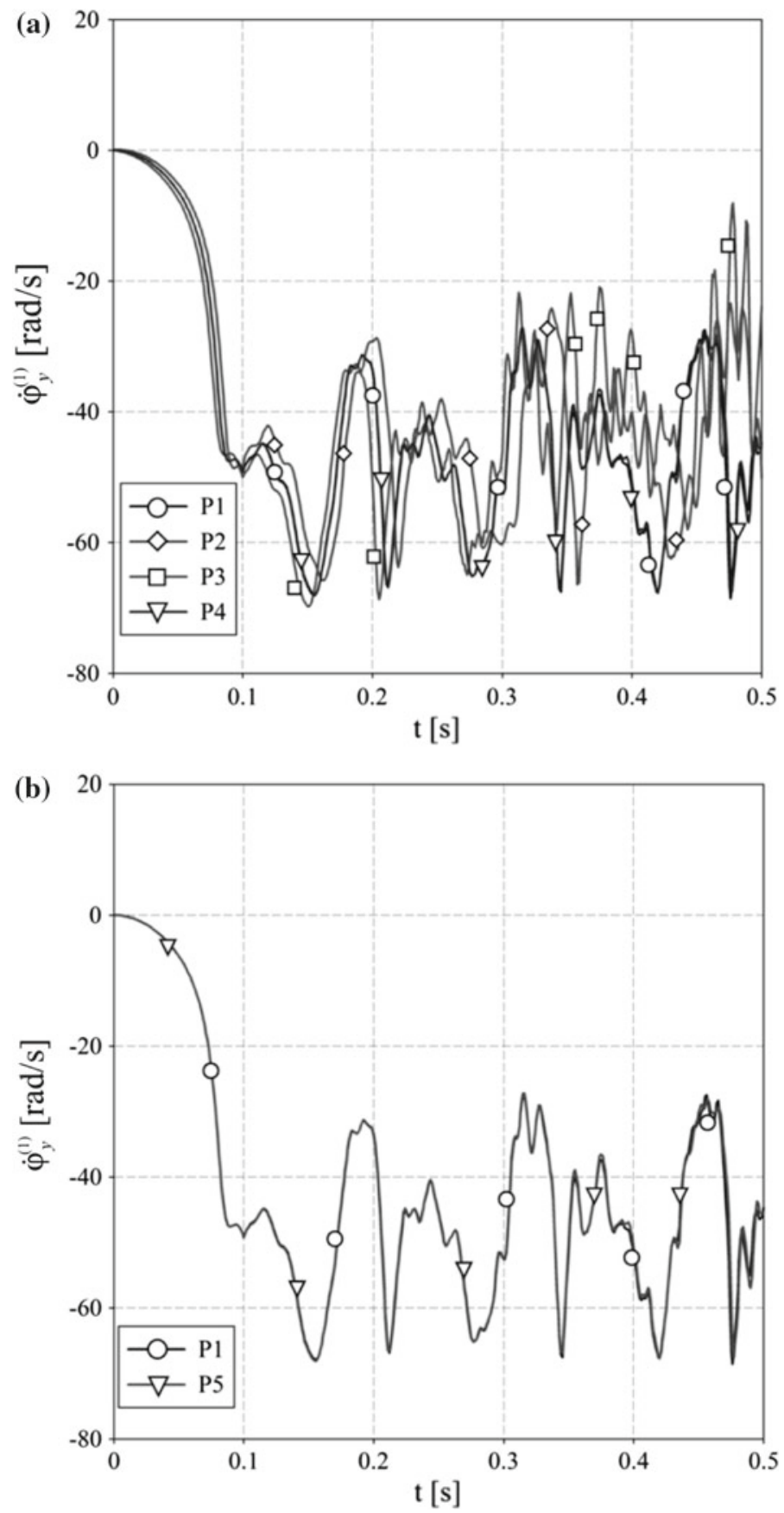

Fig. 8 Courses of angular velocity of the crank

After the necessary transformations, the kinetic energy of $\operatorname{rfe}(p, i)$ takes the form:

$$
E^{(p, i)}=\frac{1}{2} \operatorname{tr}\left\{\dot{\mathbf{B}}^{(p, i)} \mathbf{H}^{(p, i)} \dot{\mathbf{B}}^{(p, i) T}\right\}
$$

where $\mathbf{H}^{(p, i)}=\int_{m(p, i)} \tilde{\mathbf{r}}^{(p, i)} \tilde{\mathbf{r}}^{(p, i)^{T}} \mathrm{~d} m$ is a pseudo-inertial matrix.

Potential energy $V$ of the mechanism is the sum of spring energy of flexible links and energy of gravity forces: 


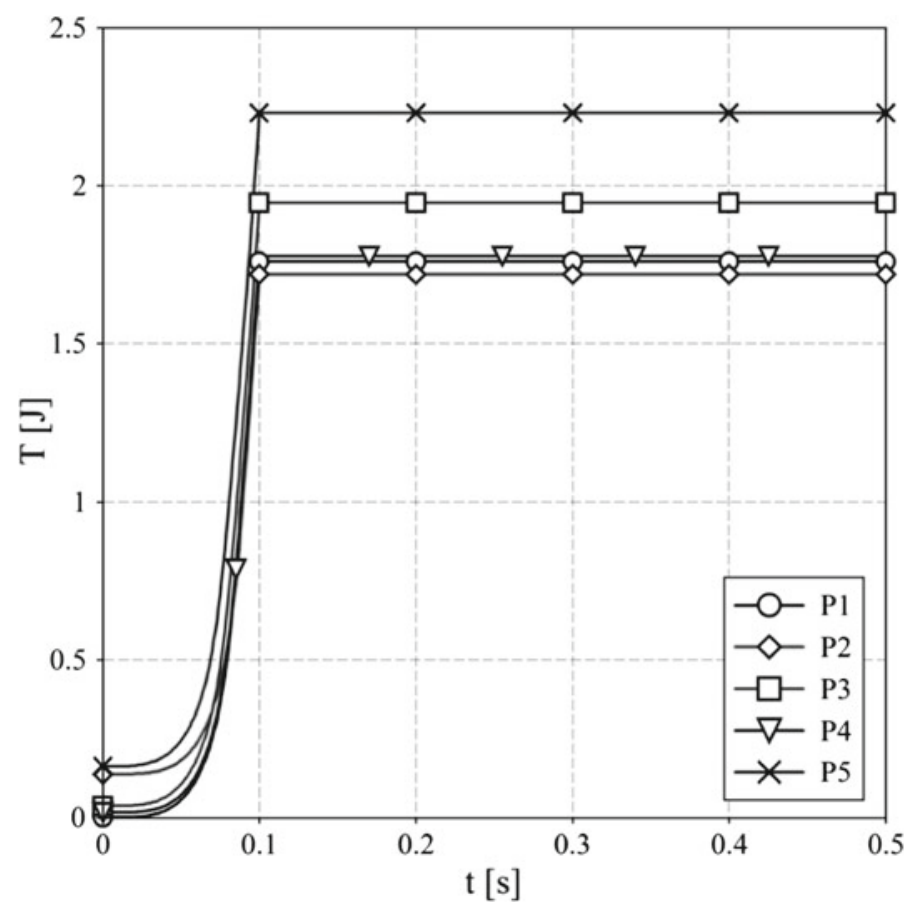

Fig. 9 Courses of total mechanical energy of the mechanism

$$
V=\sum_{p=1}^{N} \sum_{i=1}^{m^{(p)}} V_{s}^{(p, i)}+\sum_{p=1}^{N} \sum_{i=0}^{m^{(p)}} V_{g}^{(p, i)}
$$

where $V_{g}^{(p, i)}=m^{(p, i)} g z_{c}^{(p, i)}$ is the potential energy of gravity forces of $\mathrm{rfe}(p, i), m^{(p, i)}, z_{c}^{(p, i)}$ are mass and coordinates of the centre of mass of $\operatorname{rfe}(p, i)$ with respect to global coordinate system \{\} and $V_{S}^{(p, i)}$ is the energy of spring deformation of $\operatorname{sde}(p, i)$.

Equations of motion (10) have to be completed with constraint equations of the joint at which the closed kinematic chain has been cut:

$$
\boldsymbol{\Phi}(\mathbf{q})=\mathbf{0}
$$

Let us consider a rotary joint connecting $\operatorname{rfe}\left(p, m^{(p)}\right)$ with $\operatorname{rfe}(p+1,0)$ (Fig. 5). A coordinate system is assigned to each rfe in such a way that $\hat{\mathbf{Z}}^{(\kappa)}$ axis coincides with the axis of the joint.

Constraint equations of such a joint form five algebraic equations as follow:

$$
\boldsymbol{\Phi}(\mathbf{q}) \equiv\left[\begin{array}{c}
\Delta \tilde{\mathbf{r}}(\kappa) \\
{ }_{L^{(\kappa)}}{ }^{T}{ }_{P} \overline{\mathbf{Z}}^{(\kappa)} \\
\hat{\mathbf{X}}^{(\kappa)^{T}}{ }_{P} \overline{\mathbf{Z}}^{(\kappa)}
\end{array}\right]=\left[\begin{array}{l}
\mathbf{0} \\
0 \\
0
\end{array}\right]
$$

where $\Delta \tilde{\mathbf{r}}^{(\kappa)}$ is a vector describing relative position of the elements connected, ${ }_{L} \hat{\mathbf{X}}^{(\kappa) T},{ }_{L} \hat{\mathbf{Y}}^{(\kappa)}$ are vectors of coordinate system ${ }_{L}\{\kappa\}$ assigned to $\operatorname{rfe}\left(p, m^{(p)}\right),{ }_{P} \overline{\mathbf{Z}}^{(\kappa)}$ is a vector of coordinate system ${ }_{P}\{\kappa\}$ transformed to coordinate system $L\{\kappa\}$.

For dynamic analysis, we will use acceleration form of the constraint equations:

$$
\ddot{\boldsymbol{\Phi}}(\mathrm{q}) \equiv \boldsymbol{\Phi}_{\mathrm{q}} \ddot{\mathbf{q}}+\dot{\boldsymbol{\Phi}}_{\mathrm{q}} \dot{\mathbf{q}}=\mathbf{0}
$$

where $\boldsymbol{\Phi}_{\mathbf{q}}$ is a Jacobian matrix of the constraint equations. 

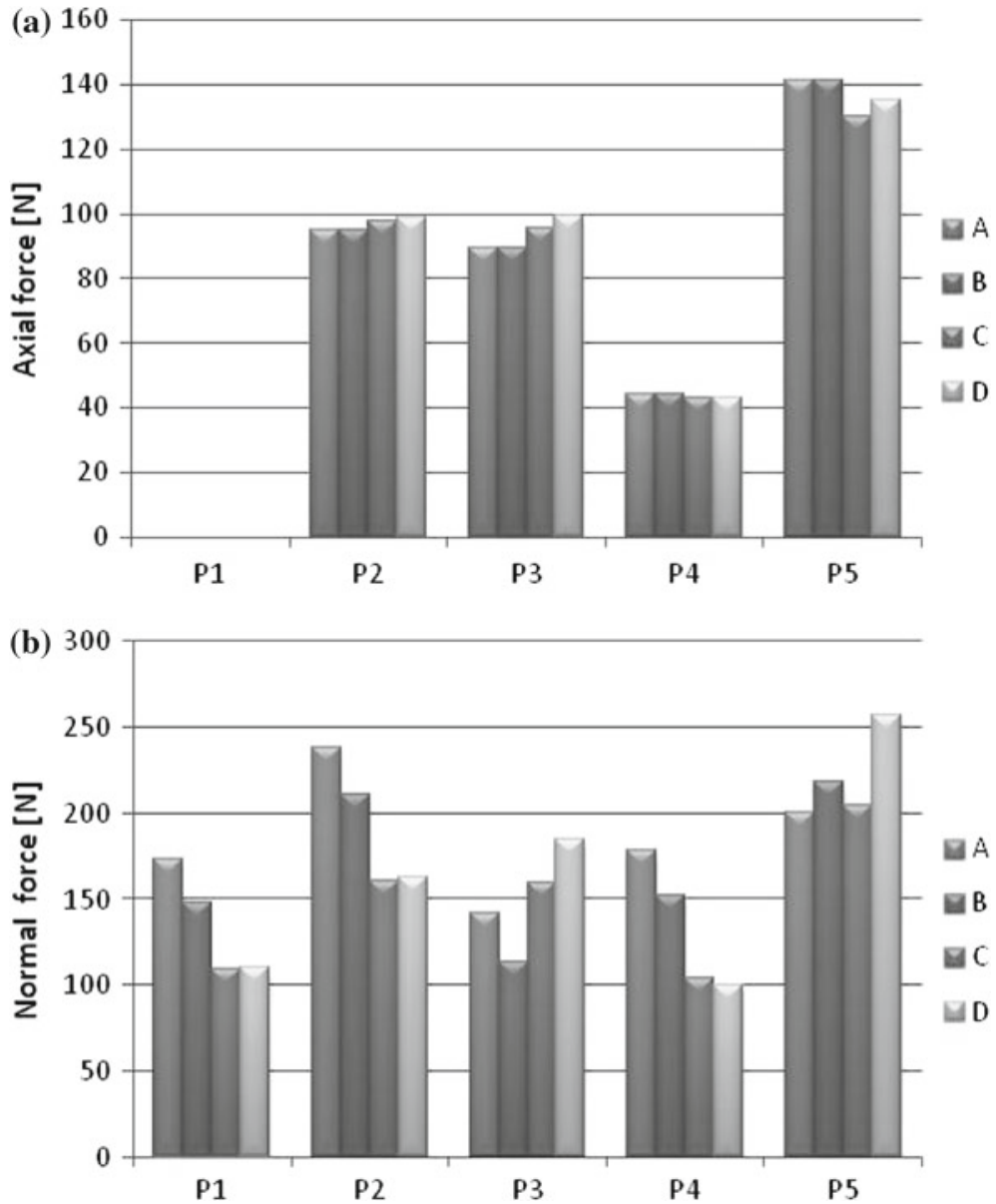

Fig. 10 Maximum axial (a) and normal (b) forces in the joints

Finally, the equations of motion can be presented in the form:

$$
\begin{aligned}
\mathbf{A} \ddot{\mathbf{q}}+\boldsymbol{\Phi}_{\mathbf{q}}^{T} \mathbf{R} & =\mathbf{F} \\
\boldsymbol{\Phi}_{\mathrm{q}}^{\ddot{\mathbf{q}}} & =-\dot{\boldsymbol{\Phi}}_{\mathrm{q}}^{\dot{\mathbf{q}}}
\end{aligned}
$$

where $\mathbf{R}$ is the vector of Lagrange multipliers related to constraint equations, $\mathbf{F}$ is the vector of generalized forces that results from dynamic forces, spring deformation forces and external loads, $\mathbf{A}=\left[\begin{array}{ll}\mathbf{A}^{L} & \mathbf{0} \\ \mathbf{0} & \mathbf{A}^{R}\end{array}\right]$ is the mass matrix of the system, and $\mathbf{A}^{L}, \mathbf{A}^{R}$ are mass matrices related to the left and right parts of the cut chain.

Equation (20) forms a set of differential-algebraic equations of index 1. The Baumgarte stabilisation method [3] can be used in order to reduce influence of constraints violation while integrating the equations of motion.

\section{Numerical simulations}

A computer program in $\mathrm{C}++$ has been worked out in order to implement the mathematical model of the mechanism. Numerical simulations have been carried out, and the four-bar linkage mechanism presented in Fig. 6 has been analysed. For the analysis, the crank has been assumed to be rigid while the connecting rod and rocker arm are flexible. Data are taken from Ref. [2]. As a result of assembly errors, the joint connecting the rocker arm with the base has been shifted and turned.

The motion of the mechanism is caused by the following moment:

$$
M^{(1)}= \begin{cases}M_{0} \frac{t}{t_{0}}, & t \leq t_{0} \\ 0, & t>t_{0}\end{cases}
$$



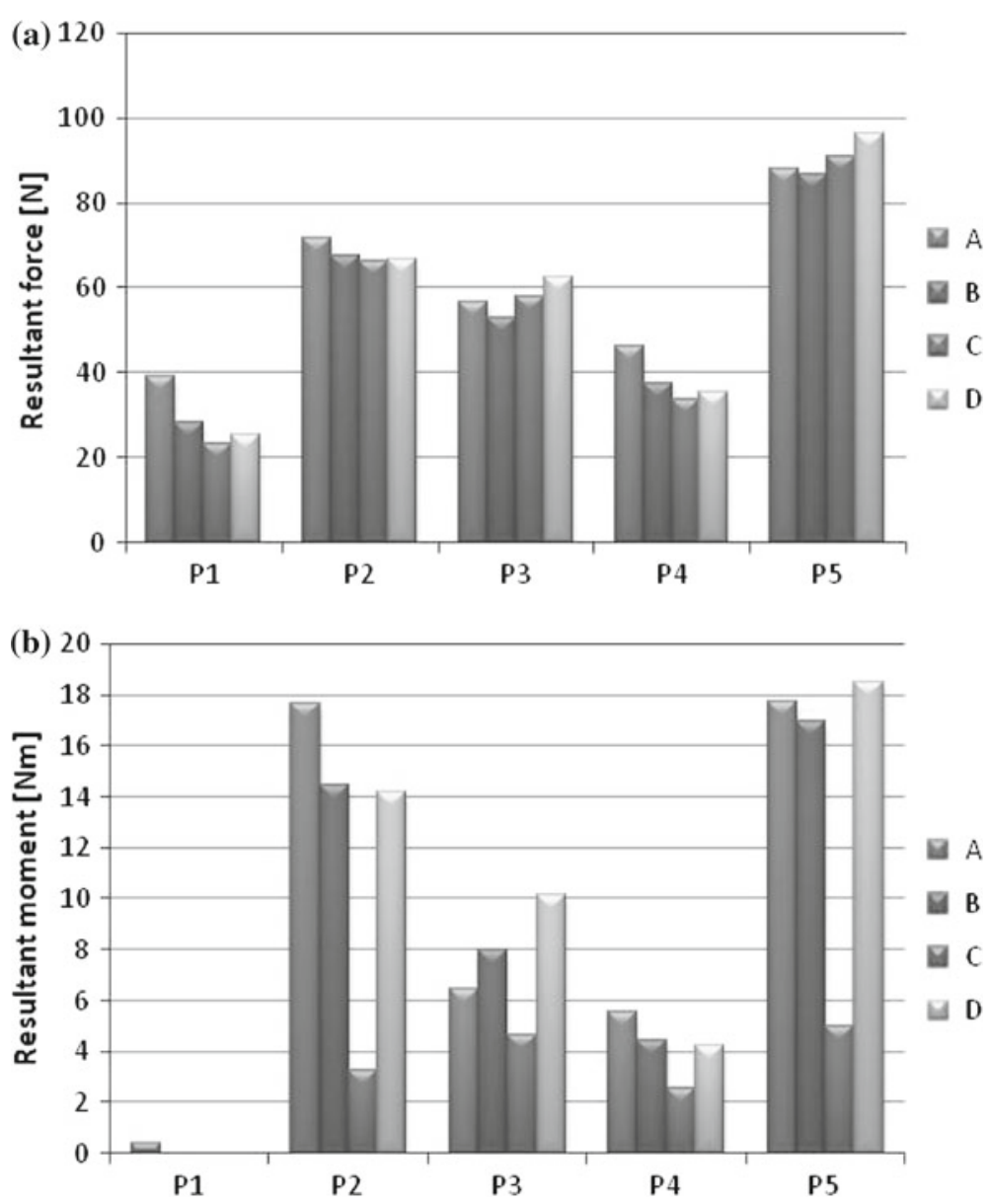

Fig. 11 RMS of resultant reaction forces (a) and moments (b) in the joints of the mechanisms

where $M_{0}$ is the moment acting at $t_{0}$ time.

For numerical simulations, it is assumed that $M_{0}=1.5(\mathrm{Nm})$ and $t_{0}=0.1(\mathrm{~s})$. The closed kinematic chain is cut at joint $C$, and reaction forces and moments are calculated with respect to the local system of the joint, using a modified Newton-Euler algorithm described in Refs. [1,4,9]. The connecting link and rocker arm are divided into 5 rfes. The calculations are carried out for the errors assumed in Table 1 .

The equations of motion were integrated using fixed step size integrator Runge-Kutta of the fourth order. The simulation time is 0.5 second, and the integration step size is $10^{-5}$. Rotation angle and angular velocity of the crank obtained from simulations are presented in Figs. 7 and 8.

It can be seen that assembly errors caused by shifting joint $\mathrm{D}$ have negligible influence on the motion of the crank. Figure 9 presents time history of the total mechanical energy of the system during the simulation. The energy becomes larger and larger when the moment acts on the crank, and it is constant after $0.1 \mathrm{~s}$.

Maximum axial and normal forces in joints $A, B, C, D$ for the cases analysed are presented in Fig. 10.

It can be stated that error $\delta_{x}$ resulting from the rotation of joint $D$ about $\hat{\mathbf{X}}^{0}$ axis (case P2) and superposition of all errors (case P5) have the largest influence on maximum forces acting in the joints. In order to measure the magnitude of the resultant forces and moments acting in the joints, root mean square value (RMS) is calculated:

$$
\begin{gathered}
F_{r m s}^{i}=\sqrt{\frac{\int_{0}^{T}\left(F^{i}(t)\right)^{2} \mathrm{~d} t}{T}} \\
M_{r m s}^{i}=\sqrt{\frac{\int_{0}^{T}\left(M^{i}(t)\right)^{2} \mathrm{~d} t}{T}}
\end{gathered}
$$



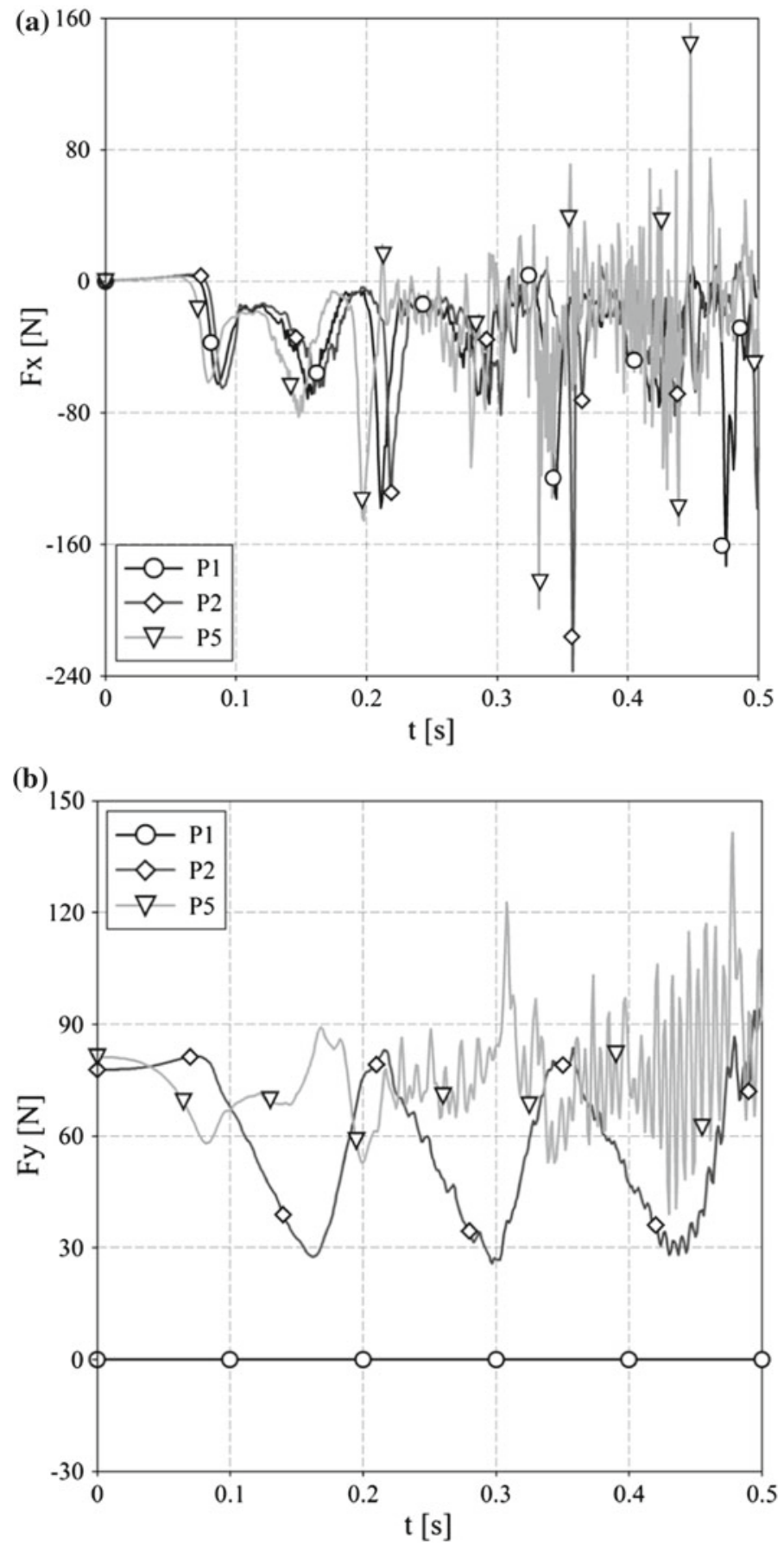

Fig. 12 Reaction forces in joint $A$ with respect to the local coordinate system

where $F_{r m s}^{i}, N_{r m s}^{i}$-resultant force and moment in joint $i, i \in\{A, B, C, D\}, F^{i}(t), M^{i}(t)$-courses of resultant force and moment, $T$ - time of simulation.

Results are presented in Fig. 11.

The results confirm that joints of the mechanisms are mostly loaded in cases P2 and P5. Change in orientation of joint $D$ has larger influence on forces and moments acting in joints than its translation. For all cases, the largest forces occurred in joints $A$ and $D$. Time history of forces acting in joint $A$ without and with assembly errors is presented in Fig. 12. 
The results shown in Fig. 11 enables us to analyse reaction forces caused by:

- external moment (case P1),

- external moment and assembly errors (cases from P2 and P5 for $t \in\langle 0 ; 0,1\rangle$ )

- only assembly errors (cases P2 and P5 for $t \in\langle 0,1 ; 0,5\rangle$ ).

\section{Final remarks}

In the paper, a mathematical model of the mechanism with flexible beam-like links, rotary joints and assembly errors is presented. Joint coordinates and homogenous transformations are used for description of motion of the system. Flexible links of the mechanism are discretised by means of the classical rigid finite element method. This method differs from that with absolute nodal variables, which is used by standard packages. The joint variables used here mean that the number of generalised coordinates, and therefore the number of equations of motion, has been reduced to a minimum. Moreover, the rigid finite element method used for modelling the flexible links can be treated as a particular case of a multibody system, and therefore, the flexibility can be taken into account using a fairly minor modification of the procedure applicable to analyse systems with rigid links. In the model presented, the assembly errors are included when kinematics of the mechanism is described. An application of the Newton-Euler algorithm enables us to calculate reactions in joints without having to divide up the system into subsystems, and thus, the reaction forces can be calculated not only in the "cut" joint. Numerical simulations allow us to analyse the influence of inaccurate assembly on the load of joints. It can be concluded that errors that change the orientation assumed considerably influence the motion of the mechanism and the loads on its links. Extension of the model presented to multiloop mechanisms with non-ideal joints will be a subject of further research.

Open Access This article is distributed under the terms of the Creative Commons Attribution Noncommercial License which permits any noncommercial use, distribution, and reproduction in any medium, provided the original author(s) and source are credited.

\section{References}

1. Adamiec-Wójcik, I.: Modelling Dynamics of Multibody Systems, Use of Homogenous Transformations and Joint Coordinates. Lambert Academic Publishing, Saarbrücken (2009)

2. Adamiec-Wójcik, I., Augustynek, K.: Modelling dynamics of flexible multibody systems by means of the rigid finite element method, In: MULTIBODY DYNAMICS ECCOMAS Thematic Conference. Madrid, Spain (2005)

3. Blajer, W.: Elimination of constraint violation and accuracy aspects in numerical simulation of multibody systems. Multibody Syst. Dyn. 7, 265-284 (2002)

4. Craig, J.J.: Introduction to Robotics. Addison-Wesley, Massachusetts (1988)

5. Gronowicz, A.: Identification of the overconstrained linkages. Wrocław Technical University Press, Wrocław (1992)

6. Pott, A., Kecskeméthy, A., Hiller, M.: A simplified force-based method for the linearization and sensitivity analysis of complex manipulation systems. Mech. Mach. Theory 42, 1445-1461 (2007)

7. Wang, S.-X., Wang, Y.-H., He, B.Y.: Dynamic modeling of flexible multibody systems with parameter uncertainty. Chaos, Solitons Fractals 36, 605-611 (2008)

8. Wittbrodt, E., Adamiec-Wójcik, I., Wojciech, S.: Dynamics of Flexible Multibody Systems: Rigid Finite Element Method. Springer, Berlin, Heidelberg, New York (2006)

9. Wojciech, S.: Dynamic analysis of manipulators with consideration of dry friction. Comput. Struct. 6, 142-153 (1995) 